\title{
Multimodal vs Unimodal Estimation of Sympathetic-Driven Arousal States
}

\author{
Sandya Subramanian ${ }^{1,2}$, Emery N. Brown ${ }^{1,2}$, Riccardo Barbieri ${ }^{2,3}$ \\ ${ }^{1}$ Massachusetts Institute of Technology, Cambridge, USA \\ ${ }^{2}$ Massachusetts General Hospital, Boston, USA \\ ${ }^{3}$ Politecnico di Milano, Milan, Italy
}

\begin{abstract}
Estimation of sympathetic-driven arousal state (SDAS) traditionally consists of computing frequency-based measures of heart rate variability. However, in the presence of confounds such as breathing frequency, these measures can incorrectly estimate the underlying SDAS. In this work, we present an example of such a case during a three-stage paced breathing task.

Using a state space framework, we demonstrate that a unimodal model that relies solely on these frequency-based heart rate variability measures overestimates SDAS during the slowest breathing stage and underestimates it in subsequent stages. On the other hand, a multimodal model with both time and frequency domain heart rate variability observations as well as electrodermal activity information provides a more realistic estimate of SDAS throughout the task. This suggests that multimodal estimation of SDAS is more accurate and robust than unimodal estimation.
\end{abstract}

\section{Introduction}

Estimation of sympathetic-driven arousal state (SDAS) is an important problem in many conditions, including pain, stress, anxiety, depression, and sleep. Traditionally, frequency-based metrics of heart rate variability computed from the electrocardiogram (ECG) are used [1-2]. The most common of these are power in low $(0.04-0.15 \mathrm{~Hz})$ and high $(0.15-0.4 \mathrm{~Hz})$ frequency ranges (LF and HF respectively). $\mathrm{HF}$ has been shown to correlate with parasympathetic tone, while LF is thought to be a combination of sympathetic and parasympathetic tone. Other derived measures are also used, such as the ratio $\mathrm{LF} / \mathrm{HF}$ or normalized values of LF and HF (LFu and HFu).

However, the unimodal nature of these indices can make them misleading, particularly in situations where the breathing rate causes respiratory sinus arrhythmia (RSA) to be a confounding factor. We hypothesized that a multimodal approach to SDAS estimation would yield more accurate and robust results. Previous attempts at multimodal autonomic state estimation did not explore the effects of breathing rate confounds [3-5]. To test our hypothesis, we collected multimodal data from five healthy volunteers during a paced breathing task. The first and slowest stage of breathing during the task was at a frequency that falls directly within the LF range.

We used a state space framework to estimate the underlying SDAS. We hypothesized that the SDAS should decrease with deep, slow breathing and increase with breathing rate. Our results show that a unimodal model based solely on LF and HF derived observations estimates the highest SDAS at the slowest stage of breathing due to the confound of the breathing rate. On the other hand, a multimodal model correctly estimates a low SDAS at the slowest breathing stage which then increases with breathing rate. These results suggest that multimodal estimation of SDAS is a more accurate and robust representation of the underlying information, especially in situations with possible confounds.

\section{Data and Methods}

In this study, we collected data from five healthy volunteers between the ages of 22 and 34 with approval from the Massachusetts Institute of Technology Institutional Review Board. The subjects were asked to perform a paced breathing task in which they had to breathe at each of three rates for three minutes each. The slowest breathing rate was $0.1 \mathrm{~Hz}$ (6 breaths per minute), then $0.2 \mathrm{~Hz}$ (12 breaths per minute), and the final stage was at $0.3 \mathrm{~Hz}$ (18 breaths per minute). We collected continuous ECG, respirations (using a deflection sensor around the torso), and electrodermal activity (EDA) data throughout the task. EDA measures the change in conductance of the skin due to sweat gland activity, which is purely sympathetically controlled [6]. Three of the subjects had a two-minute baseline period before the start of the task.

We then computed a series of features from the collected data. Using a point process heart rate variability model, we computed instantaneous estimates of mean and standard deviation of heart rate, as well as frequency domain measures of heart rate variability such as LF/HF and $\mathrm{HFu}[7-8]$. We also filtered the EDA data into a slow- 

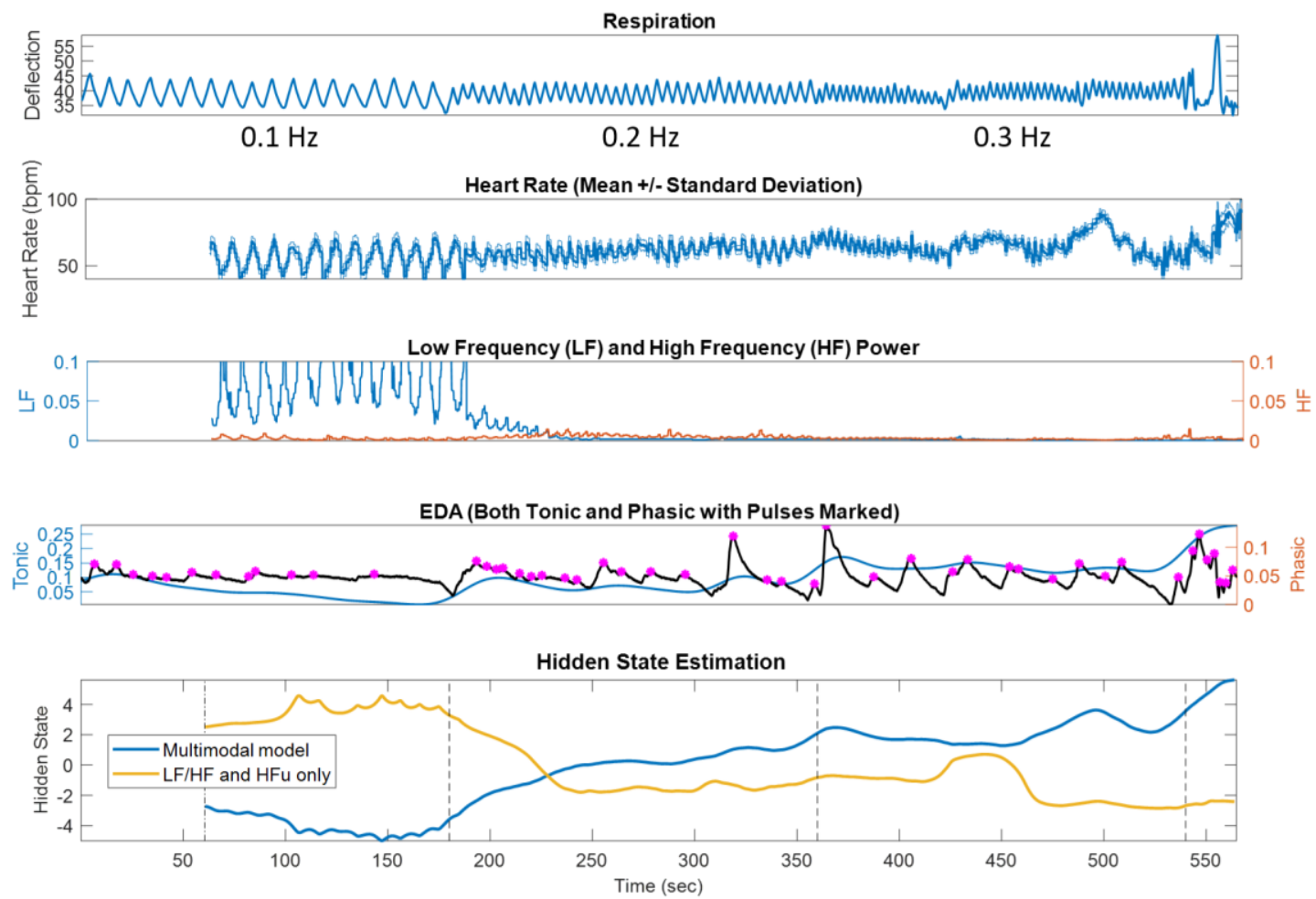

Figure 1. Subject 1 Paced Breathing Task results, showing (from top to bottom) respirations, instantaneous mean heart rate \pm standard deviation, instantaneous estimate of low and high frequency power (with separate y-axes), tonic and phasic EDA activity with pulses labeled, and the sympathetic-driven arousal state estimates in a state space model with multimodal versus unimodal observations. Vertical lines in bottom panel separate the three breathing stages of the task.

moving tonic or baseline component and a pulsatile phasic component from which we extracted pulses. The details of EDA processing can be found in [9].

Table 1. Summary of six observations for each subject.

\begin{tabular}{ll}
\hline Observation & Details of Computation \\
\hline Mean heart rate & $\begin{array}{l}\text { Point process HRV model, } \\
\text { averaged for each window }\end{array}$ \\
$\begin{array}{l}\text { Standard deviation } \\
\text { of heart rate }\end{array}$ & $\begin{array}{l}\text { Point process HRV model, } \\
\text { averaged for each window } \\
\text { LF/HF }\end{array}$ \\
Point process HRV model, \\
averaged for each window \\
HFu & HFu = HF/(LF+HF) \\
& $\begin{array}{l}\text { Point process HRV model } \\
\text { Averaged for each window }\end{array}$ \\
Tonic EDA & $\begin{array}{l}\text { Low pass filter on EDA data } \\
\text { Averaged for each window }\end{array}$ \\
Phasic EDA & $\begin{array}{l}\text { Pulses extracted from phasic EDA } \\
\text { In each window, either 0 (if no } \\
\text { pulse) or pulse amplitude (if there } \\
\text { is a pulse) }\end{array}$ \\
\hline
\end{tabular}

We took the mean of each feature after normalization in non-overlapping 0.5 second windows as observations for a state space model. For phasic EDA, observations were either zero or a pulse amplitude within each window. The six observation time series are summarized in Table 1.

We then applied a linear Gaussian state space approach to estimate the underlying SDAS. This framework models SDAS evolution over time as an autoregressive process, where all of the observations depend only on the underlying state at each time point. The state evolution and observation equations of the model are below.

$$
\begin{gathered}
\text { State Evolution Equation: } \\
x_{k}=A+B x_{k-1}+\varepsilon_{x}, \quad \varepsilon_{x} \sim N(0, Q) \\
\text { Observation Equation: } \\
Y_{k}=E+F x_{k}+\varepsilon_{Y}, \quad \varepsilon_{Y} \sim N(0, R)
\end{gathered}
$$

We tested two models, one with all six observation time series, and one with only LF/HF and HFu as observations. We used the expectation maximization algorithm to simultaneously estimate the underlying state over time and the parameters of the model $(A, B, E, F, Q, R)$. This algorithm consists of a forward filter and backward smoother to estimate the underlying state and maximum likelihood estimation of the model parameters. Finally, the estimates of the underlying state evolution over time from both models were compared. 

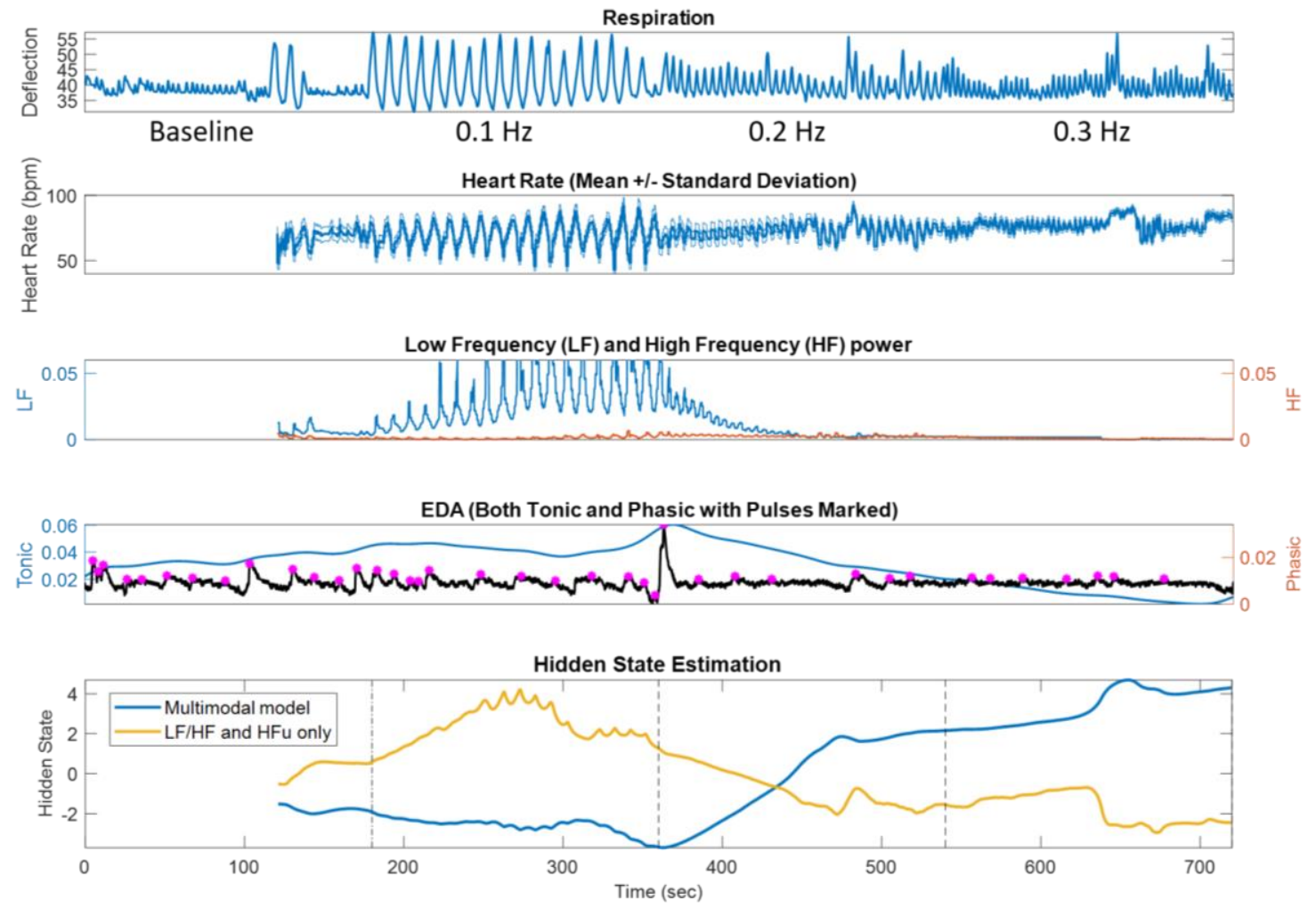

Figure 2. Subject 2 Paced Breathing Task results. See Fig. 1 for details. This subject had an additional baseline period before the start of the task.

\section{Results}

Looking at the data (Figs. 1 and 2), the respirations clearly delineate the three stages of the task, including the baseline beforehand for Subject 2. While the mean heart rate oscillates noticeably due to RSA, there is no clear trend in terms of increasing or decreasing heart rate across the three stages of the task. This also holds true for the standard deviation of the heart rate. The RSA is most pronounced in the slowest stage of breathing.

Because the breathing frequency of the first stage of the task $(0.1 \mathrm{~Hz})$ falls squarely in the range of $\mathrm{LF}$, it is strongly affected by the RSA and mimics the same oscillatory pattern with high amplitude during the first stage of the task. While HF also seems to have some oscillations throughout, they are much smaller in amplitude. Finally, there seems to be no consistent trend in either the tonic or phasic EDA across the different stages of the task.

Looking at the SDAS estimates for individual subjects and across all five subjects (bottom panel of Figs 1 and 2, Fig 3), the two models consistently give very different estimates of the SDAS throughout the task. The unimodal model, which uses only LF and HF derived features as observations, estimates the SDAS of the first stage of the task to be high, higher than that of baseline even. This initial overestimate, which is clearly influenced strongly by the effect of RSA on LF, is also much higher than the SDAS estimate in subsequent stages of the task. In contrast, the multimodal model estimates a very low SDAS during the first stage of the task, decreasing from baseline. Then the SDAS estimate increases through the second and third stages of the task, with most of the increase happening in the second stage.

\section{Discussion}

In this study, we analyzed multimodal autonomic signal data from five subjects during a paced breathing task. We used a state space framework to estimate the underlying SDAS, but using two different models. In the multimodal model, observations included both HRV and EDA measures, but in the unimodal model, only frequencybased HRV metrics were observations. Because the breathing rate of the slowest stage of breathing fell within the frequency range of one of the HRV metrics, the unimodal model estimates the highest SDAS during the slowest breathing stage of the task. The multimodal model, on the other hand, estimates a low SDAS during this stage which then increases in subsequent stages. This is in better agreement with known physiology associated with deep, slow breathing.

Slow, deep breathing is a core component of many stress relief practices such as yoga and meditation, as well 


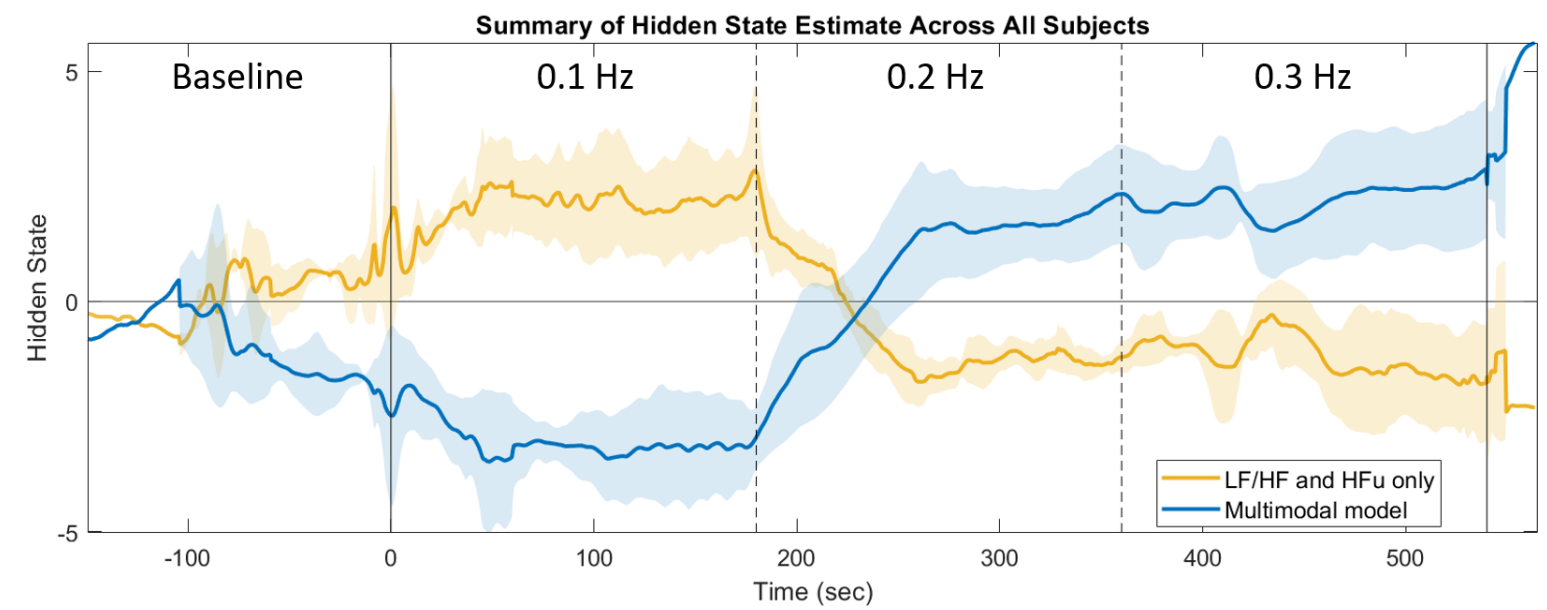

Figure 3. Summary of sympathetic-driven arousal state estimates across all subjects shows as the mean \pm standard deviation. The start and end of the task are at 0 and 540 seconds respectively and the stages are separated with dashed vertical lines.

as a recommended therapeutic strategy for anxiety or panic attacks. In all of these cases, the goal is to drive down sympathetic activation. In addition, studies have shown that guided breathing exercises increase vagal activity and decrease blood pressure and peripherical sympathetic nerve activity [10]. Therefore, it is reasonable to conclude that the multimodal model provides a more accurate estimate of the true SDAS as being low during the first stage of the task, especially compared to normal baseline level of activity. The unimodal model not only overestimates the SDAS during the first stage of the task, it biases the SDAS for the other two stages of the task due to the frequency-dependent buffering of RSA [11].

It is noteworthy that the multimodal model is able to overcome influence of RSA on LF at the slowest stage of breathing based on combined information from the other observations and even in the absence of a clear trend in any of them. This supports the use of multimodal signals to inform SDAS estimation, especially when there is no information about breathing rate. Future work will include refining the observation model in the state space framework and adding more modalities of information, such as blood pressure or pulse oximetry. In addition, this multimodal paradigm will be tested in other settings, including pain, anesthesia, and sleep.

\section{Acknowledgments}

S.S. would like to thank the MIT Clinical Research Center staff.

\section{References}

[1] S Akselrod, D Gordon, FA Ubel, DC Shannon, RJ Cohen. Power spectral analysis of heart rate fluctuation: a quantitative probe of beat-to-beat cardiovascular control. Science 213, 220-223 (1981).
[2] G Valenza, L Citi, JP Saul, R Barbieri. Measures of sympathetic and parasympathetic autonomic outflow from heartbeat dynamics. Journal of Applied Physiology (2018).

[3] S Ghiasi, A Greco, R Barbieri, et al. Assessing autonomic function from electrodermal activity and heart rate variability during cold-pressor test and emotional challenge. Sci Rep 10, 5406 (2020).

[4] S Ghiasi et al. A new sympathovagal balance index from electrodermal activity and instantaneous vagal dynamics: a preliminary cold pressor study in Proceedings of the 40th IEEE International Conference on Engineering in Medicine and Biology. (2018).

[5] DS Wickramasuriya, RT Faghih. A mixed filter algorithm for sympathetic arousal tracking from skin conductance and heart rate measurements in Pavlovian fear conditioning. PLOS ONE 15(4): e0231659 (2020).

[6] W Boucsein, Electrodermal Activity. (Springer), (2012).

[7] R Barbieri, E Matten, A Alabi, E Brown, A point-process model of human heartbeat intervals: new definitions of heart rate and heart rate variability. Am. J. Physiol. Hear. Circ. Physiol. 288, H424-435 (2005).

[8] R Barbieri, E Brown, Analysis of heartbeat dynamics by point process adaptive filtering. IEEE Transactions on Biomed. Eng. 53, 4-12 (2006).

[9] S Subramanian, R Barbieri, EN Brown, A point process characterization of electrodermal activity in Proceedings of the 40th IEEE International Conference on Engineering in Medicine and Biology. (2018).

[10] B Oneda, K Ortega, J Gusmão, et al. Sympathetic nerve activity is decreased during device-guided slow breathing. Hypertens Res 33, 708-712 (2010).

[11] JP Saul, RD Berger, P Albrecht, SP Stein, MH Chen, RJ Cohen. Transfer function analysis of the circulation: unique insights into cardiovascular regulation. American Journal of Physiology. 1991;261:H1231-1245.

Address for correspondence:

Sandya Subramanian

77 Massachusetts Ave. 46-6057

Cambridge, MA 02139

sandya@mit.edu 